\title{
Internet of Things of Trees - Conversational Objects via SMS Protocols
}

\author{
Martin de Jode ${ }^{1}$, Leah Lovett ${ }^{1}$, Duncan Hay ${ }^{1}$, Andy Hudson-Smith', Richard Milton ${ }^{1}$, Lucy \\ Fraser $^{2}$ \\ ${ }^{1}$ Bartlett Centre for Advanced Spatial Analysis, UCL, Gower Street, London, UK \\ ${ }^{2}$ City of London Corporation, Guildhall, London, EC2P 2EJ, UK
}

Keywords: INTERNET OF TREES, SMS, GEOFENCING, HAMPSTEAD HEATH

\begin{abstract}
The paper describes a 'work in progress' to develop a system to enable users to engage with the historical and environmental story behind veteran trees in Hampstead Heath in the spirit of the Internet of Things. Unlike other 'Internet of Trees' projects, this study focuses on story telling rather than sensor networks. Building on previous work, conversational agents ('chatbots') are used as proxies for the trees to enable a two-way narrative exchange between the user and the 'tree'. Two interaction pathways are proposed (direct SMS and web-based geofencing) and the technical development of both approaches is described, as well as ethnographic studies undertaken on Hampstead Heath to elicit engaging content for the chatbot. An initial deployment of the SMS-based interaction at Tate Exchange, a project space within Tate Modern, London is discussed, and a preliminary evaluation presented.
\end{abstract}

\section{Introduction}

There has been considerable interest on the application of Internet of Things (IoT) technologies for story telling systems exemplified by projects such as Tales of Things [1] and Tales of the Park [2]. In this paper we describe a work in progress to apply similar technologies to telling the stories around trees, particularly focused on Hampstead Heath, London. The application of IoT technologies to arboreal environments is not new [3]. However, previous work has focused on the deployment of sensor networks to determine and react to the physical or biological environment. Instead, here we aim to explore different aspects including the historical, environmental and cultural stories behind some of the prominent trees in Hampstead Heath.

\section{Background}

The approach taken builds on previous work undertaken as part of PETRAS, in which a number of IoT objects, embedded with Bluetooth Low Energy (BLE) beacons and backed by a chatbot, were deployed around the Queen Elizabeth Olympic Park, London. Tales of the Park enabled the public to converse via a web application that could be accessed from their mobile browser. This approach obviated the need to install an application ('app') thereby removing a significant barrier to engagement, but relied on Google's Physical Web [4], that was at the time supported by the Google's Chrome browser that is installed by default on Android phones (and can be installed on iOS devices). The Physical Web project provided a way for mobile devices to interact with BLE beacons. However, in late 2017 Google withdrew support for the Physical Web from their Chrome browser, and thereby rendered the approach we adopted in Tales of the Park obsolete. Hence this has necessitated the exploration of alternative forms of interaction. 


\section{Methodology}

\subsection{Approach}

In this project we continue the theme of trying to maximise accessibility to the IoT through mobile platforms whilst avoiding the need to install apps. The Internet of Things of Trees explores two approaches to the problem. The first involves development of web application that exploits geofencing capabilities in HTML 5 and the fact that trees are stationary. Again, this is an entirely browser-based approach and has no requirement to install apps. Conscious that even a web-based interaction presents some barriers to adoption (as well as requiring a smart-phone and sufficient data, the user has to start the browser, point it to the appropriate URL and ensure location tracking is enabled to make this approach viable), we also explore an even more direct approach through the use of SMS to interact with the trees, removing the need for the user to possess even a smart-phone.

Both approaches focus on a text-based interface, employing conversation agents (chatbots) to simulate a natural language interaction. To enable the SMS interaction mode an SMS gateway is required. The function of an SMS gateway is to convert SMS messages on the mobile cell network to Internet Protocol (IP) messages and vice versa. The SMS gateway enables conventional internet-based software systems to access the content of SMS messages via a webservice. Once the chatbot has generated a text response, this is also delivered by the SMS gateway over the cell network to the recipient, via SMS. For our application it is necessary to hook up the chatbot to the SMS gateway so that the chatbot receives the text message and delivers the response.

The geofencing web application is simpler in that text messages can be delivered directly to the chatbot via the Internet (provided the user is in the vicinity of the tree).

The use of both interfaces simultaneously creates further opportunities for a comparative methodology, specifically exploring the relative accessibility and adoption of IoT Technologies within a public space (Hampstead Heath).

\subsection{Ethnographic Study and Content Development}

In parallel to the technical development, an ethnographic study is being undertaken on Hampstead Heath with a view to uncovering the social and cultural significance of the fifteen trees included in the project. The project trees were initially selected by the City of London arborists who manage the Heath to include 'veterans' across a range of native and imported species, all of them remarkable either for their age, historical significance, or ornamental value, and often for a combination of these reasons. A series of interviews, drop-in arts workshops and guided walks with the arborists, visitors to the heath and local community groups have provided the space for these stakeholders to share their own stories around the trees. These ethnographic methods have been combined with archival research into the natural and social history of the trees and the Heath in general to produce a corpus of stories from which to develop engaging chatbot content.

The corpus is input into jGnoetry [7], a web-based program that processes existing text to generate original poetry. This platform was chosen above alternative n-gram generators (e.g. CharNG, eDiastic) because it enables a particularly high degree of user interaction, resulting in a process of human-computer co-authorship. Another advantage of this method from an ethics/GDPR perspective is that it effectively anonymises any personal data that may have been gathered during the ethnographic research.

The poetic fragments resulting from processing the found stories through jGnoetry were subsequently grouped according to keywords (e.g. 'vertigo', 'storm', 'aground' etc) linked to each of the fifteen project trees to provide engaging content for the chatbot.

\subsection{Data Protection Challenges}

The decision to employ SMS as an interaction modality presents certain challenges from a data privacy perspective since it is necessary for the system to process the user's mobile phone number, which constitutes personal identifiable data under current data protection regulations. The initial intention was to employ a thirdparty SMS gateway provider. A suitable service was identified that, although US-based, was EU-US Privacy 
Shield compliant. To minimise personal data our system would not retain user telephone numbers, however the third-party gateway provider would need to store the user's phone number for a period (120 days) for the purposes of billing us (not the user) for the service.

In accordance with university policy we submitted an application to the university data protection office for data protection registration. After some discussion we were advised that, as the SMS Gateway provider represents a third-party data processor located outside the EU, it would be necessary for a GDPR compliant 'controller to processor' legal agreement to be in place between the university and the third party. It was suggested that a more straightforward route would be to explore options that did not involve the processing of personal data outside the EU. In light of this advice and given the short timescales of the project we consequently decided to develop and deploy our own 'inhouse' SMS Gateway solution.

\subsection{Building the SMS Gateway}

It is relatively simple to build a small-scale SMS gateway by making use of the AT ('Attention') command set [5] supported by many GSM compatible phones. AT commands are text commands that are sent to the phone over the serial interface and allow control of a number of telephony related functions including sending and receiving text messages over SMS.

A small Java application was written that made use of the open source SMS Library v3 [6] to simplify access to the AT command set. A Wavecom GSM Modem (plus SIM card) was used as the telephony hardware (although a Sony Ericsson k750i mobile phone was also found to work well). The Java application was hosted on a Raspberry Pi which connected to the GSM modem over the USB serial interface (see Figure 1).

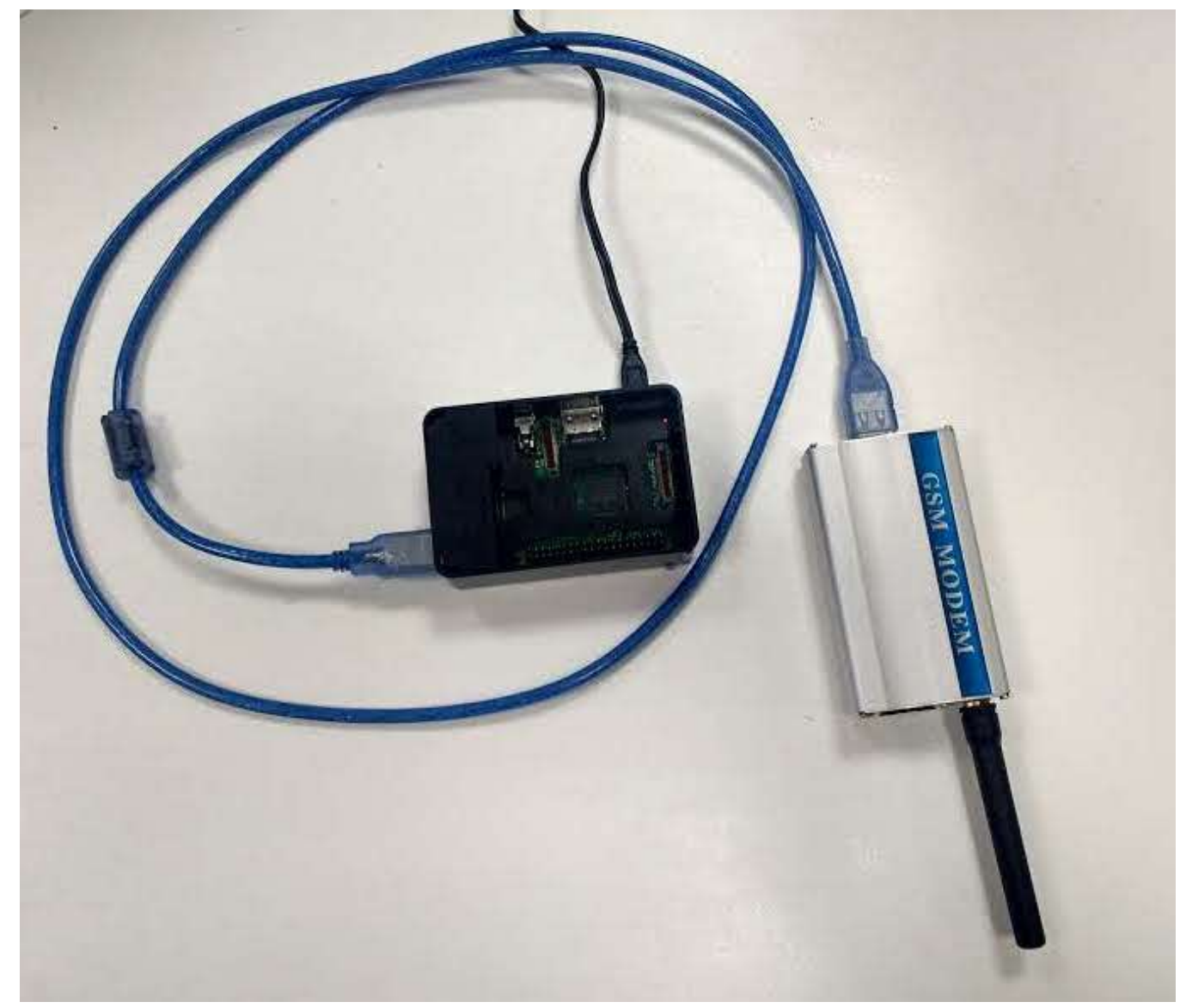

Fig. 1 A simple SMS gateway was constructed utilising a GSM modem and a Raspberry Pi which links incoming SMS messages to the DialogFlow chatbot. 
The operation of the SMS interaction mode is as follows:

i) The user sends an SMS message to the GSM gateway (i.e. the phone number of the SIM card in the GSM modem).

ii) The Java application pulls the message off the GSM modem via the AT command set.

iii) The Java application extracts the message body from the text message and forwards it to the DialogFlow chatbot (dialogflow.com) over a web service.

iv) The chatbot interprets the text using natural language processing and sends a suitable response to the Java application.

v) The Java application constructs a return text message using the chatbot response and uses the AT command set to cause the GSM modem to return the text message to the recipient over SMS.

vi) The Java application stores the message body in a small database but deletes the SMS message from GSM modem so that the user's phone number is not stored. The most obvious downside of using a 'homemade' SMS gateway was a limitation on the scalability of the system, as each phone number deployed required its own GSM telephony device.

\subsection{Building the Web Application}

As an alternative to the SMS interaction mode, a geofencing web application was developed. This makes use of the geolocation capability introduced in HTML 5 and now supported by modern browsers. A backend database was prepopulated with the geolocation of the 15 trees selected by the Hampstead Heath arborists. The user is able to use a browser-based map to navigate around Hampstead Heath (see Figure 2) and when their location is within 15 metres of one of these trees a simple chat dialog will automatically open enabling the user to send a text message over the Internet to the chatbot representing the tree. The web application database is also used to record all text messages (whether transmitted via the web application or SMS gateway via a simple custom API). 'The Listening Wood' was selected as a suitable name for the project and registered as a domain name.

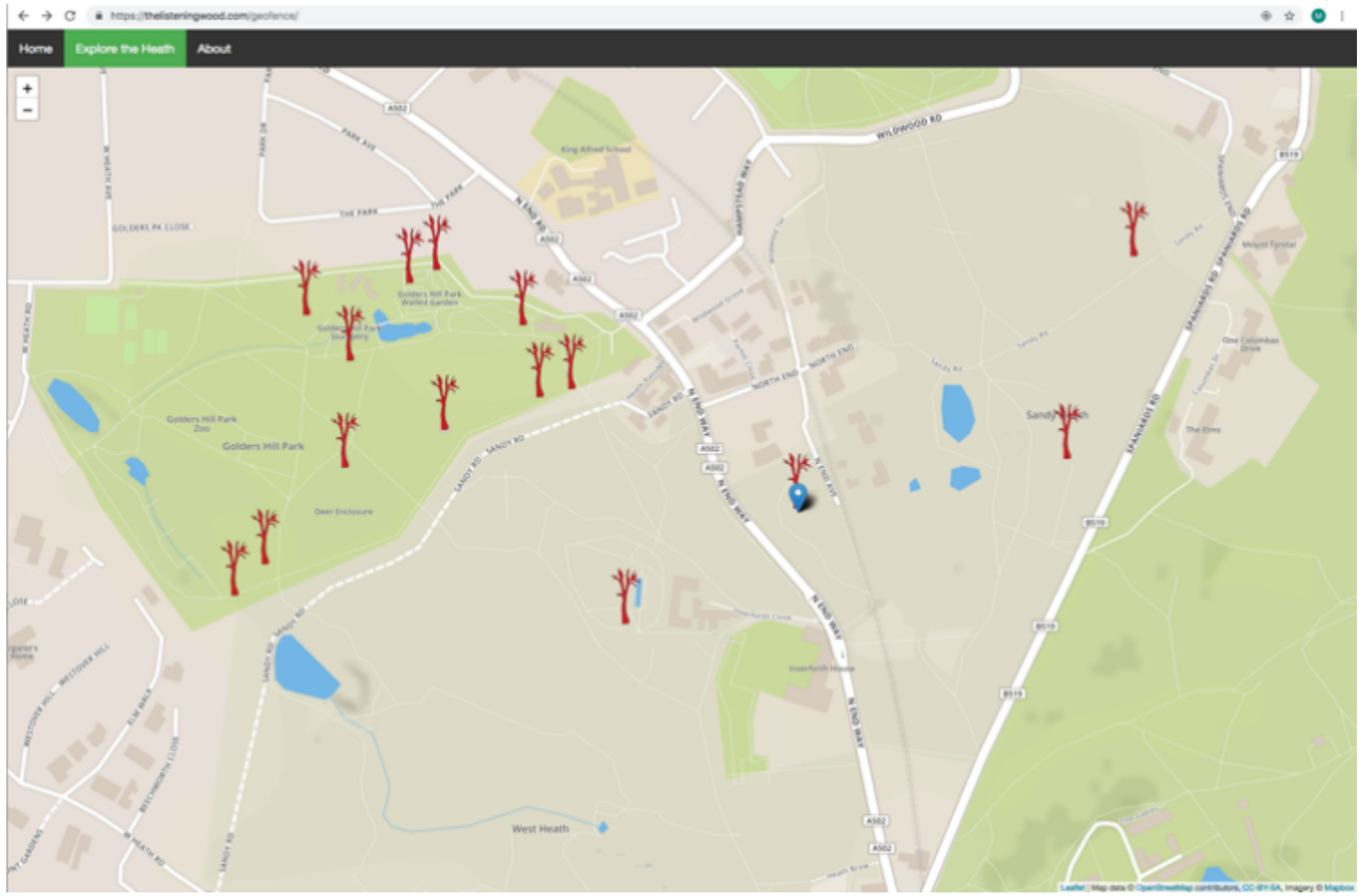

Fig. 2 Prototype geofencing web application for the Hampstead Heath deployment (thelisteningwood.com) 


\section{Results}

\subsection{Deployment at Tate Modern}

An initial deployment of the SMS system was undertaken as part of the PETRAS end of grant exhibition at the Tate Modern. Entitled 'The Listening Wood', this iteration of the project incorporated five out of the fifteen project trees, represented as a series of large-format photographs printed onto fabric and suspended from the gallery ceiling. Each image was hung between wooden battens, onto which the keywords and SMS phone number for the Listening Wood had been laser-cut. On sending an SMS message containing the keyword to the given number, visitors received a line of poetry connected to that tree and informed by its history and the stories that surround it. The use of keywords displayed above each image enabled us to link specific narratives to individual trees, while using a single phone number.

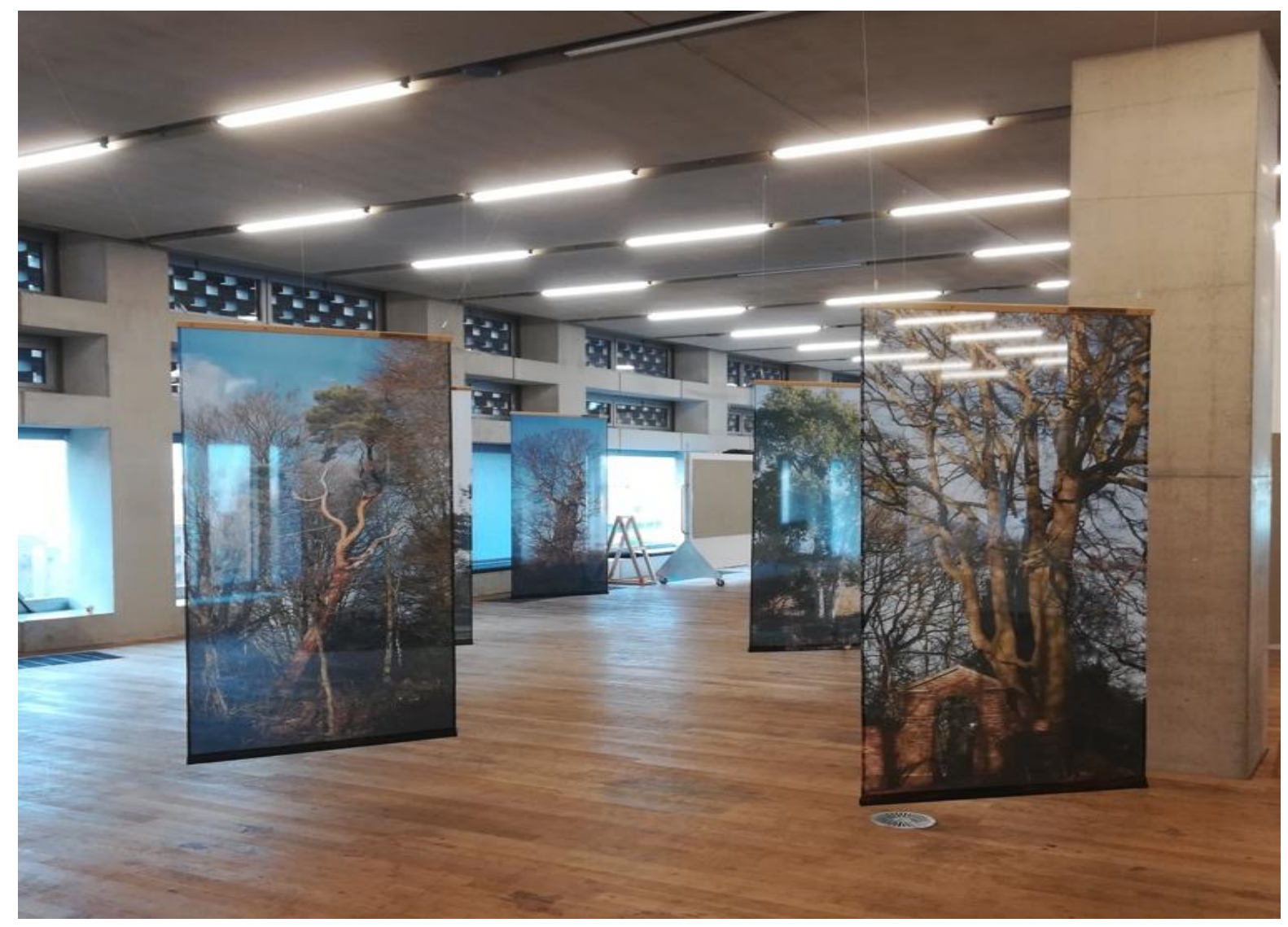

Fig. 3 Deployment at the Tate Exchange space as part of the PETRAS 'Living with the Internet of Things' event, 8-9th February 2019, Tate Modern, London.

The installation at the Tate Exchange space proved to be a success both from an artistic and technical standpoint. The suspended prints provided an impressive visual impact that drew favourable comments from both visitors and gallery curators. The SMS interaction mode proved reliable and effective, with some 450 text messages being received by the system during the two-day event. Typical round-trip response times (from sending the message to receiving the response) were 10-15 seconds, which although not as fast as a commercial SMS gateway, were more than adequate. Occasionally response messages were delayed by a greater period (as is possible with a datagram-based service like SMS that offers no QoS guarantees), but this was a rare event. Most visitors were happy to engage with the system, although one or two were reluctant to participate ("why would I text a tree") which is a perfectly understandable viewpoint. An unexpected bonus from our decision to employ our own SMS gateway, was that visitors appreciated only having to text one phone number to access the trees (our original plan was to have a phone number per tree but had to be revised when use of a commercial 
third-party SMS gateway proved impractical). Overall the installation was deemed a considerably success and gave confidence for future deployments.

\subsection{Deployment at Hampstead Heath}

The deployment 'in the wild' of the final system at Hampstead Heath took place at the end March 2019. The keywords for the selected trees were laser-cut onto slices of wood supplied by the arborists and embedded in the ground adjacent to the appropriate tree (see Fig. 4). An information leaflet was distributed explaining the project and including the phone number for SMS interaction. At the time of writing, some 6 days after the launch, about 100 messages had been received by the system, split 50/50 between the SMS interaction mode and the geofencing website. Feedback from the launch event was positive and the technology generally worked well, although the effectiveness of the geofencing web-based interaction mode seemed somewhat dependent on the model of smartphone used.

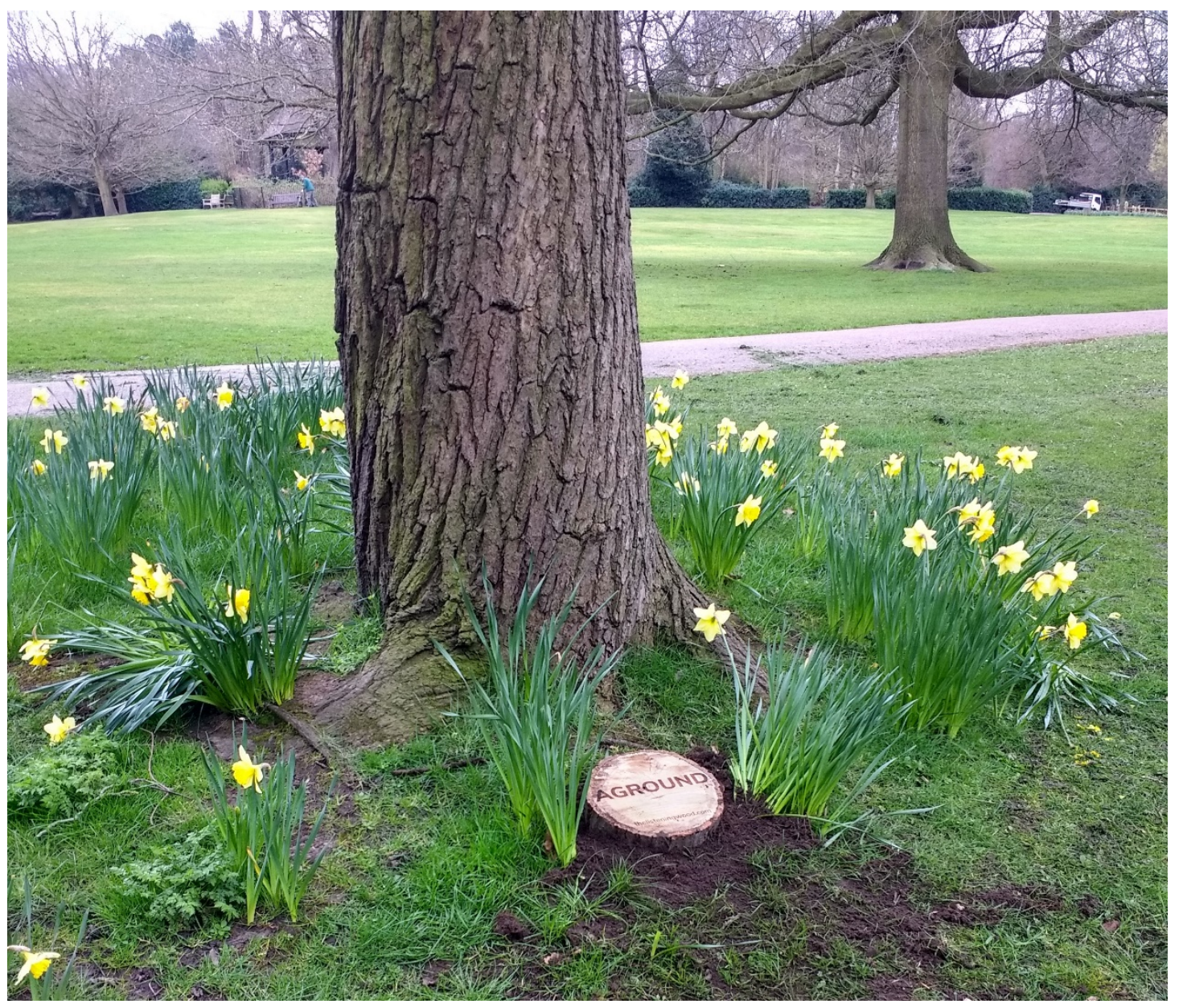

Fig. 4 Deployment 'in the wild' at Hampstead Heath, London, showing the keyword laser-cut onto a wood roundel.

\section{Conclusion}

Although a still work in progress this study aims to further develop the use of Internet of Things technologies for narrative and text-based systems and the use of conversational agents to provide a natural language interface to the content. In an initial deployment of the SMS pathway at the Tate Exchange, the system was found to work well technically and visitors engaged enthusiastically. The SMS interaction mode proved to be very accessible for users and most were happy to trust the system with their phone number, perhaps reassured by the nature of the project and the event. 
Deployment of the full system on Hampstead Heath took place in late March 2019 and is planned to run until August 2019. Initial feedback was positive and the technology worked well. Further evaluation will be undertaken when more results from this deployment become available.

One interesting challenge that arose from the development of the SMS interaction mode was the impact that the GDPR has had on the ability to use third-party cloud services based outside of the EU. In this study we worked around the issue by deploying our own SMS gateway. However, the requirement for bespoke ControllerProcessor agreements to be in place to access such cloud services has the potential to impact many future research projects.

\section{Acknowledgements}

The authors would like to thank the EPSRC for funding this research through the Privacy, Ethics, Trust, Reliability, Acceptability and Security (PETRAS) grant (EP/N02334X/1), Cyber Security of the Internet of Things and the IoT in the Park projects. We would also like to thank the City of London Corporation and the Hampstead Heath arborists for their support throughout the project and also the Tate Exchange and their team who assisted us with the installation of the first iteration of the project.

\section{References}

[1] Barthel R, Leder Mackley K, Hudson-Smith A, Karpovich A, de Jode M, Speed C. An internet of old things as an augmented memory system. Personal and ubiquitous computing. 2013 Feb 1;17(2):321-33.

[2] Milton R, Buyuklieva B, Hay D, Hudson-Smith A, Gray S. Talking to GNOMEs: Exploring Privacy and Trust Around Internet of Things Devices in a Public Space. In: Extended Abstracts of the 2018 CHI Conference on Human Factors in Computing Systems 2018 Apr 20 (p. LBW632). ACM.

[3] Internet of Trees. [online]. Available at: http://internetoftrees.io [Accessed: 28 Nov. 2018].

[4]'TODAY-Google stops supporting the Physical Web on iOS', [online] available at: https://github.com/google/physical-web/issues/931 [Accessed: 7 Feb. 2019].

[5] 'Hayes command set', [online] available at: https://en.wikipedia.org/wiki/Hayes_command_set [Accessed: 7 Feb. 2019].

[6] SMSLib v3 by Thanasis Delenikas [online] available at: https://github.com/tdelenikas [Accessed:7 Feb 2019].

[7] jGnoetry [online] available at: http://www.eddeaddad.net/jGnoetry [Accessed: 8 Feb. 2019]. 